\title{
IMPLEMENTATION OF LINK-BASED ENTERPRISE DMS SUPPORTING ISO 9001 QMS: CASE STUDY
}

\author{
DOI: 10.12776/QIP.V17I2.241
}

\author{
MAREK KAČMÁR
}

\section{INTRODUCTION}

The purpose of this paper is to describe a process of development and implementation of a Document Management System (DMS) supporting ISO 9001 Quality Management System (QMS) of an enterprise organization based on the real life example. DMS as a core element of ISO 9001 QMS, has not only to fulfill the basic ISO 9001 requirements, such as identification, formatting, review, approval and proper dissemination of documents, but it has to be accommodated to the company's environment and culture in order to be effective and sustainable.

The paper provides a review of similar papers focused on DMS implementation, describes available methodology and applies it in the case study. It provides an overview of results from individual phases of a 6-step implementation method and describes fundamental elements of a DMS. It defines key performance indicators for the monitoring and continuous improvement of the document management process and explains their use. In general the paper provides useful information and lessons learnt for any researchers involved into design, implementation, maintenance and continuous improvement of a DMS.

\section{TERMINOLOGY AND METHODOLOGY}

Document management seems to be a well-known topic within an enterprise environment, however it sometimes lacks a common understanding of what it actually is. "Document management has evolved to the point where a 'document' can be virtually any sort of a computer file" (Veal, 2001). High variety of different types of documents, their purposes and plenty of different requirements to manage these documents lead to different understanding of the document management objectives.

Following tag crowd (Figure 1), created from more than 100 abstracts of selected journal articles, represents an academic common understanding of the "document management". 


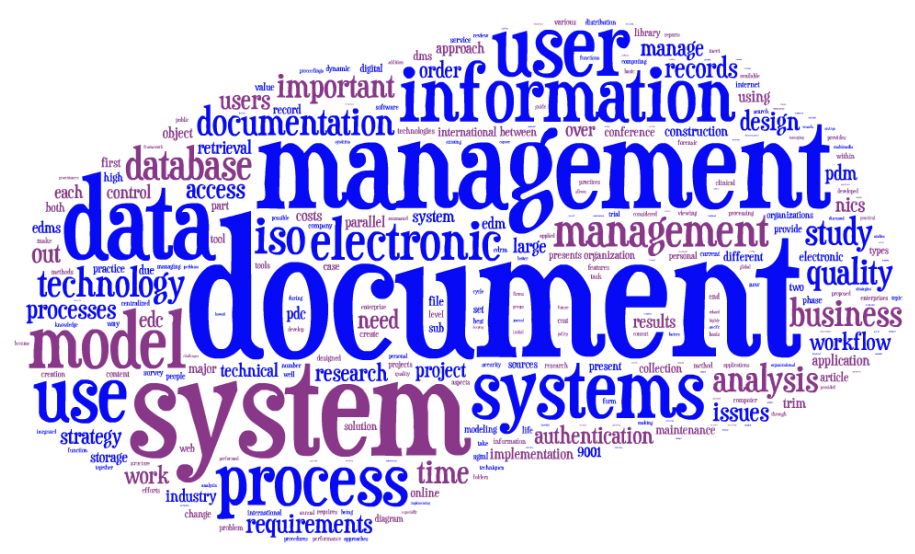

Figure 1 - Academic understanding of document management

Within a business organization, a document and its information usually represents a company's asset. The type of the information and its sensitivity, importance or urgency lead to different requirements and strategies how to manage these documents. Document management has to ensure an effective and efficient application of the strategies and fulfilment of the requirements.

\subsection{Document Management according to ISO 9001}

One typical application of the document management, which will be discussed further within this article is a DMS supporting ISO 9001 Quality Management System.

DMS is a key part of a Quality Management System based on ISO 9001, which defines several mandatory and optional documents, which companies, striving for the ISO 9001 certification should create, use and further maintain. According to ( ISO/TC 176/SC 2/N 525R2, 2008) the most important of these documents are:

- Quality Policy \& QMS manual

- Few mandatory procedures (control of documents, control of records, control of preventive and corrective actions, internal audits, etc.)

- other documents for effective planning, operation \& control of organization's processes

According to (ISO 9001, 2008) the main requirements on the control of the documents of the QMS are:

a) to approve documents for adequacy prior to issue,

b) to review and update as necessary and re-approve documents,

c) to ensure that changes and the current revision status of documents are identified, 
d) to ensure that relevant versions of applicable documents are available at points of use,

e) to ensure that documents remain legible and readily identifiable,

f) to ensure that documents of external origin are identified and their distribution controlled, and

g) to prevent the unintended use of obsolete documents, and to apply suitable identification to them if they are retained for any purpose.

\subsection{Terminology defined prior to implementation}

It became visible early during first steps of the implementation, that proper definitions of some terms are missing. ISO 9001 defines the term document and record but in reality, both can have form of paper-based or electronic documents. In order to minimize the confusion during the implementation, following definitions of different types of documents have been developed:

\section{Controlled document}

Document which is subject to change and contains essential information, therefore it is important to ensure its control in order to fulfill specified requirements. Examples are:

- Policy, Procedure, Work instruction, Manual;

- Plan, Specification;

- Template, Checklist, Form.

\section{Record}

Record is any electronic or paper-based document created during the lifecycle of a business process, sometimes in a form of an evidence that a certain activity has been performed correctly. It is created at a specific date and usually is not a subject of further change. Examples are:

- meeting protocol, filled checklist or form;

- contract, agreement;

- Record of the knowledge (within the knowledge management platform).

\section{Uncontrolled documents}

Any other documents without the need of a control, e.g. drafts, personal notes.

\subsection{Literature review}

There are several journal articles dealing with the topic of implementation of electronic DMS in the enterprise, exploring different characteristics and objectives of the document management. Gilani points to the fact that however there are computer based systems to manage the documents, they still involve physical paper and he is developing a paperless model of a document management within the university (Gilani, Ahmed, \& Abbas, 2009). Petra writes about the implementation of a DMS using the tool Sherlock, developed by 
European House of Quality $\mathrm{A} / \mathrm{S}$ in Denmark. He goes through the ISO requirements on the document management and document control and demonstrates how to use the tool in order to fulfil them (Petra, 2013). Summers provides a brief overview of web technology and possibilities to use corporate intranet and SGML based documents for the purposes of a DMS (Summers, Chelsom, Nurse, \& Kay, 1996). Tarng and Liu write about creation of a DMS within the governmental office and they are providing interesting best practices and strengthening the facts that's that up to $25 \%$ of managerial time is spent on the document retrieval and also that DMS is a part of an organizational culture and therefore it must suit also the needs of the users (Tarng \& Liu, 1994). Another article describes the recent need of Chinese companies for certification of management systems and is focusing on the development and implementation of own tool based on J2EE (Sui, Shi, Song, \& Yuan, 2008).

\subsection{Methods used in the case study}

Further in the article there is used a methodology for implementing a DMS which has been defined based on the study of "ISO9001:2008 Quality Management Systems" and "ISO 30301:2011 Information and documentation - Management systems for records" and consists of six steps:

1) Definition of document requirements;

2) Evaluation of existing systems;

3) Identification of document management strategies in the organization;

4) Design the Document Management System;

5) Implementation of the Document Management System;

6) Maintenance and continuous improvement of the Document Management System (Hernad \& Gaya, 2013).

Additionally in Step 5. Implementation, has been divided into following 4-step process in order to apply the document management strategy on a specific group of documents within an organization:

Identify - identification of key processes, documents, their sources and existing good practices for document management.

Analyse - categorization and classification of the documents into groups and their evaluation.

Define - registration of the valid documents into the system.

Control - automated control of the further document lifecycle.

Documentation of the DMS implementation is supported with the qualitative correlation \& evaluation table on Figure 3, which is used through the case study to collect individual requirements, information about the situation before the implementation and perform a correlation with application features to identify 
important design specifications. Table also helps during post-implementation review to summarize the findings and define potential improvement actions.

\section{CASE STUDY: DOCUMENT MANAGEMENT IMPROVEMENT}

Organization we will be dealing with in this case study is a large $(>1000$ employee) production unit of an international corporation (>10000 employee) providing E2E services to customers worldwide. Growth of the business over past years has risen much faster than the development of the requirements and strategies to manage the documents required for the business itself. Standardized products and tools, but various specific service differences, led to plenty of electronic documents, ranging from different forms, styles and overall quality, residing on various electronic document storage systems. Lack of control mechanisms applied and missing document management culture caused that the state of the existing system constituted a risk for planned ISO 9001 recertification.

Document Management Improvement (DMI) has been initiated as a formal company-wide project, reporting regularly to the top management in order to achieve appropriate attention to the progress of the project and to obstacles it has to face in order to be successful.

Objective of the project: Improve the Document Management process and implement it in the entire company, fulfilling the objectives of ISO 9001.

Scope of the project: redesign of the DMS for the documents required by QMS, e.g. policies, instructions, manuals etc., implementation of the DMS, training of the employees

Out of scope of the project: update of the content of the affected documents, records management.

\subsection{Definition of document requirements}

In addition to ISO 9001, some additional requirements have been defined:

\section{Align with corporate policy and systems}

Organization is a part of an international corporation, and therefore responsible to implement the existing corporate policies and systems. Unfortunately, corporate international DMS doesn't fulfill the local requirements and therefore company must develop his own local solution, taking into consideration the most fundamental elements in order to remain aligned with the corporate policy.

\section{Enable monitoring of effectiveness}

In order to ensure successful implementation, Key Performance Indicators (KPI) of the document management process shall be defined and implemented to enable automated monitoring and analysis of the process performance. 
All requirements, similarly like outputs of all upcomming phases, have been documented in the Figure 3.

\subsection{Evaluation of existing systems}

Company used several document storage systems, wikis, shared and local drives. There has been also an official DMS used mainly for the purposes of Central Functions, but not used by the production.

DMS for central functions consisted of a simple document storage system and one central document controller who ensured that new documents and updates were in an appropriate format and submitted a paper-based protocol for a sign-off to document owner, following a 5-step process:

- Drafting - creation of a document draft;

- Reviewing - check of content and form;

- Release - approval;

- Publication - publication on DMS and information of users;

- Monitoring - monitoring of document validity.

Production on the other hand used several different systems to store and maintain a different kind of documentation, but without proper policing and control mechanisms.

Several departments started to use wiki-based solution to keep their key information and knowledge on un-managed wiki pages who almost everybody can review and update. Some departments reduced the edit rights only to specific users and some applied add-ons to enable article approval, to ensure that the user can retrieve only the officially released procedure. This kind of wiki-systems might be accepted as a QMS for the ISO 9001 QMS (Castano, Mendez, \& Day, 2012)

\subsection{Identification of document management strategies in the organization}

Main strategies defined during the improvement project were:

- use the company's already existing intranet portal adjusted with additional features, developed by internal resources,

- replace existing DMS for central functions,

- remove the use of paper and enable operation to benefit from its use as well,

- don't enlarge the central function for document control but rather distribute the responsibilities by training selected staff in departments to be able to act in the role of a document controller, 
- accept wiki-based solutions with content approval as a valid approach to manage the technical work instructions and promote that as a best practice to other departments.

\subsection{Design the Document Management System}

Based on the collected requirements, evaluation of existing systems and defined strategy, the project designed three main DMS functionalities.

\section{Link based system with metadata structure}

Link based system enables to store the documents directly but also to register only links to the documents stored on external sources, wikis, etc. Employees then can access a list of all documents, even though they are stored in different locations.

Metadata structure replaced the former folder structure, faster search results and greater variability in possibilities of presenting the different lists of documents.

\section{Approval workflow}

Simple workflow should be triggered each time a new version of a document is registered involving document controller and content manager, and optionally an owner, see Figure 2. The versioning should differentiate between two types of changes:

- Minor - small changes to content - without involvement of Owner in the approval;

- Major - changes affecting the flow of the procedure or with more significant impact on the business - with involvement of Owner in the approval.

The last approval stage by Company-Document Manager is a temporary one, and will be switched off after the process will be under control of Document Managers in individual departments.

\section{Monitoring of validity}

A background job should be running on a regular basis, compare the expiration date of the document with the current date and informs the owner and controller early in advance to update or archive document or prolong its validity further.

\subsection{Implementation}

Objective of the implementation phase has been to map the important documents in the departments and register them in the system, train the affected staff and ensure a proper handover of the responsibilities to authorized employees. 


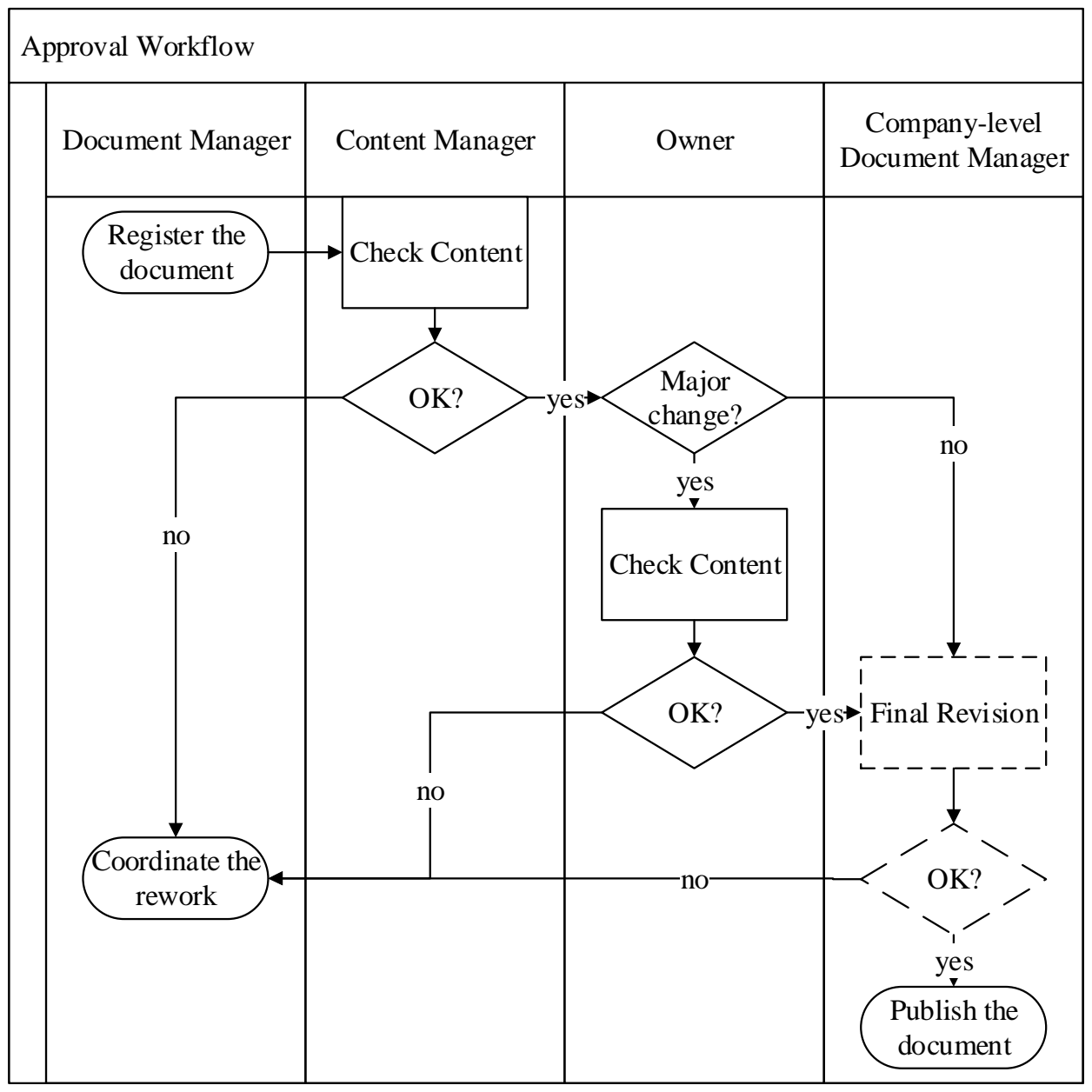

Figure 2 - Approval workflow

Prior to any further activity, an updated Document Management Policy has been published as an example of easy-to-understand controlled document describing the main activities and rules of the document management process.

\section{Identify}

The most interesting results of the first step Identify are:

- More than 10000 documents collected in over 40 departments.

- High variability from 4 to 7000 in departments suggesting significant document management culture differences .

\section{Analyse}

Documents collected in the step 1 have been further analysed and discussed with the experts. Several dominant issues become visible: 
- Significant differences in the quality of the document format from department to department - leading again to culture differences.

- Many cross-area documents identified in several departments.

- International corporate documents without a clear ownership, labelled as a draft.

- Wrong understanding of the basic definitions - even after a brief explanation, a lot of records and temporary documents have been collected in the step 1 .

\section{Define}

Finally only few documents per department have been selected to be registered in the DMS in the project round. Documents with lower importance, unknown ownership or with wrong type were left out and will be added later after postimplementation review.

\section{Control}

After the documents have been registered and approved by respective owners, they became monitored by the system itself, which sends an email notification, informing owners about the need to update the documents in regular intervals.

\subsection{Maintenance and Continuous improvement of the Document Management System}

After the implementation of the system, a post-implementation review has been conducted, to evaluate the effectiveness of the implementation and fulfilment of the original requirements. Implemented system has been reviewed from the perspective of each requirement and observations and potential follow up activities documented in Figure 3.

Following regular maintenance and continuous activities will strive to stabilize the system and improve it with new features:

- monitoring of KPIs and their analysis,

- regular review of documents in scope,

- collect ideas for improvement and regularly select tool change requests,

- planning and controlling of improvement actions. 


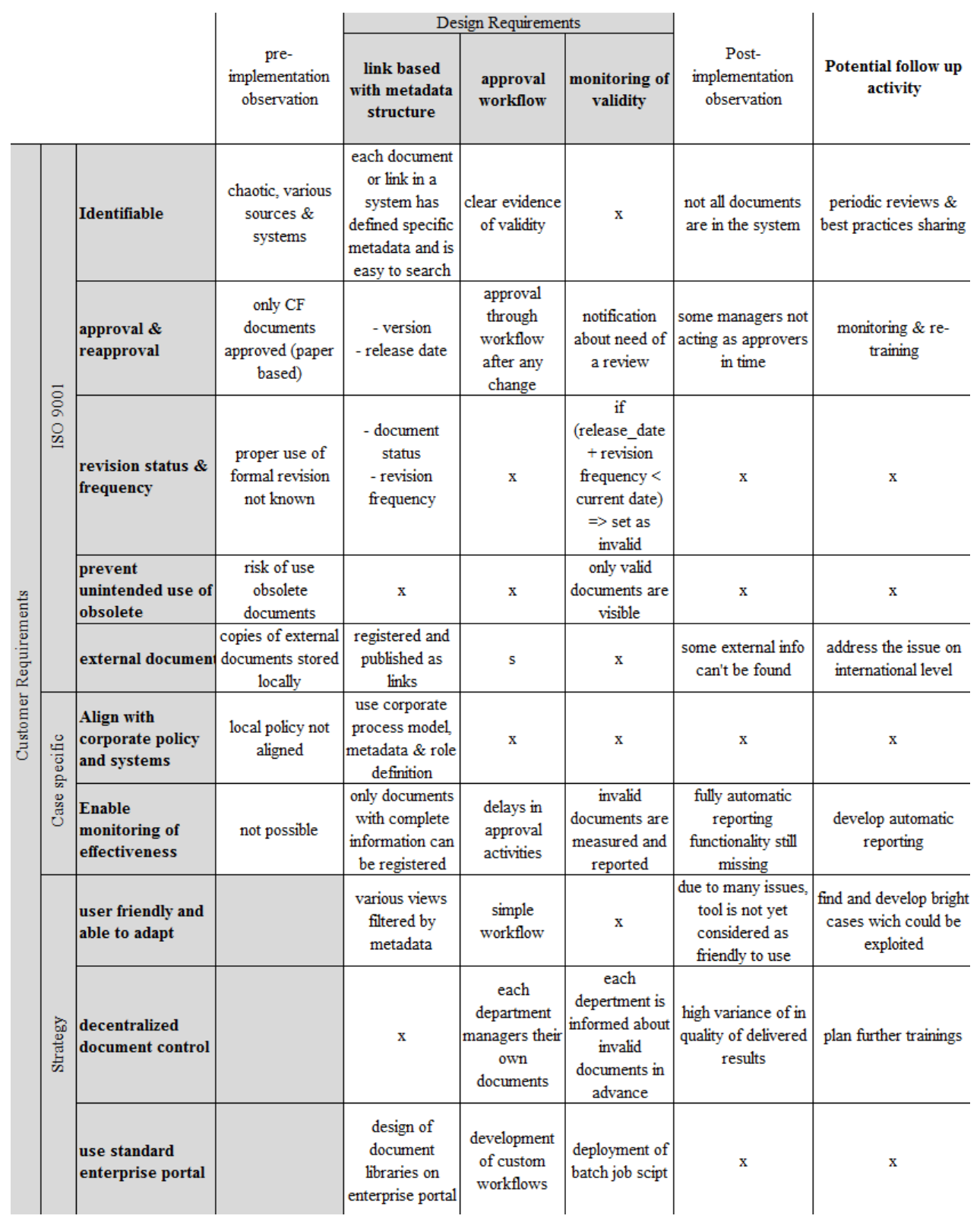

Figure 3 - Qualitative correlation \& evaluation matrix

In order to implement a monitoring of the process performance, following KPIs have been defined:

\section{Documents in scope - Number of registered documents (RD)}

Documents in scope measure simply a number of registered documents per organizational unit (e.g. department) and is a simple quantitative indicator useful for comparison with other departments and for a trend analysis. 


\section{Ratio of valid documents (RVD)}

Ratio of valid documents is based on the amount of valid documents per organizational unit and measures the overall effectiveness and compliance.

$$
R V D i=\frac{V D i}{R D i} * 100 \%
$$

Where $V D$ represents valid documents, $R D$ represents registered documents and $i$ defines scope of measurement within an organization, e.g. department.

\section{Approval success (AS)}

Approval success should measure a ratio between number of successful approval workflows (SW) per number of all workflow approvals (W) for a given organizational unit (e.g. department) and for a given timeframe (e.g. month). It should identify the departments with high number of rejected workflows due to formal errors. Documents managers of such departments should be then retrained.

$$
A S i=\frac{S W i}{W i} * 100 \%
$$

\section{CONCLUSIONS}

DMS described in this article has been successfully implemented and has been positively evaluated during the process of ISO 9001 re-certification. Link-based solution enabled the implementation of the central DMS without the need to migrate the documents or prohibit the use some of the existing document storage systems.

However, several issues surfaced during the implementation which do not enable an easy adaptation of the tool by employees. Further continuous improvement activities strengthened with appropriate communication to users are therefore important.

Further studies could be focused on the improvement of the end user interface, deeper analysis of the identified documents in order to find best practices how to document the work instructions, and on the interaction with the systems of other organizations of the corporation leading to a central corporate DMS.

\section{REFERENCES}

ISO/TC 176/SC 2/N 525R2, 2008. Guidance on the Documentation Requirements of ISO 9001:2008. Geneva: International organization for Standardization. 
Castano, F., Mendez, G., \& Day, L., 2012. Geometrica builds ISO 9001 QMS on Wiki. ASQ Knowledge Center. Available at: <http://asq.org/knowledgecenter/iso-9001-qms-wiki.html>.

Gilani, S., Ahmed, J., \& Abbas, M., 2009. Electronic Document Management: A Paperless University Model. 2nd IEEE International Conference on Computer Science and Information Technology, 8-11 Aug. 2009, Beijing, pp.440-444, DOI: 10.1109/ICCSIT.2009.5234679

Hernad, J., \& Gaya, C., 2013. Methodology for implementing Document Management Systems to support ISO 9001:2008 QMS. Procedia Engineering, Volume 63, 2013, pp. 29-35, DOI: 10.1016/j.proeng.2013.08.225.29-35.

ISO 9001, 2008. Quality Management Systems. Geneva: International organization for Standardization.

Petra, C. M., 2013. Document Management for the Implementation and Maintenance of Management Systems Case study - Sherlock Web. Quality Access to Success, Vol. 14, No. 134/June 2013, p. 80.

Sui, L., Shi, G., Song, P., \& Yuan, X., 2008. Design and Implementation of ISO Document Management System. 2008 International Conference on Computer Science and Software Engineering, 12-14 Dec. 2008, vol. 2, pp.417-419, DOI: $10.1109 /$ CSSE.2008.1343

Summers, R., Chelsom, J., Nurse, D., \& Kay, J., 1996. Document Management: an Intranet approach. In: Engineering in Medicine and Biology Society. Bridging Disciplines for Biomedicine. Proceedings of the 18th Annual International Conference of the IEEE, vol.3, Amsterdam, pp. 1236 - 1237. DOI: 10.1109/IEMBS.1996.652789.

Tarng, M.-Y., \& Liu, H.-Y., 1994. Creating a document management system. Industrial Management \& Data Systems, Vol. 94, Iss: 9, pp.9 - 15. DOI: 10.1108/02635579510101465

Veal, D. C., 2001. Techniques of Document Management: A review of text retrival and related technologies. Journal of Documentation, Vol. 57 Iss: 2, pp. 192 - 217, DOI: 10.1108/EUM0000000007082

\section{ABOUT THE AUTHOR}

Ing. Marek Kačmár - PhD. student, Technical University of Košice, Slovakia, e-mail:marek.kacmar@tuke.sk 